\title{
Modelling disorder: the cases of wetting and DNA denaturation
}

\author{
S. Ares ${ }^{1, a}$ and A. Sánchez ${ }^{2}$ \\ 1 Max Planck Institut für Physik komplexer Systeme, Nöthnitzer Str. 38, 01187 Dresden, Germany \\ 2 Grupo Interdisciplinar de Sistemas Complejos (GISC) and Departamento de Matemáticas, Universidad Carlos III de Madrid, \\ Avenida de la Universidad 30, 28911 Leganés, Madrid, Spain \\ and \\ Instituto de Biocomputación y Física de Sistemas Complejos, Universidad de Zaragoza, 50009 Zaragoza, Spain
}

\begin{abstract}
We study the effect of the composition of the genetic sequence on the melting temperature of double stranded DNA, using some simple analytically solvable models proposed in the framework of the wetting problem. We review previous work on disordered versions of these models and solve them when there were not preexistent solutions. We check the solutions with Monte Carlo simulations and transfer matrix numerical calculations. We present numerical evidence that suggests that the logarithmic corrections to the critical temperature due to disorder, previously found in RSOS models, apply more generally to ASOS and continuous models. The agreement between the theoretical models and experimental data shows that, in this context, disorder should be the crucial ingredient of any model while other aspects may be kept very simple, an approach that can be useful for a wider class of problems. Our work has also implications for the existence of correlations in DNA sequences.
\end{abstract}

PACS. 87.15.-v Biomolecules: structure and physical properties - 68.35.Rh Phase transitions and critical phenomena - 05.40.-a Fluctuation phenomena, random processes, noise, and Brownian motion

\section{Introduction}

The study of disorder in physical systems has proved fruitful not only for better understanding of complex systems, but also because new and powerful methods have been developed in order to cope with the problems posed by disordered systems. Thus, tools developed in the context of physical problems have been applied to the study of biological systems, where disorder (or, in general, inhomogeneity) is ubiquitous. In fact, inhomogeneity is frequently the most important part in a given biological function. This is the case, for instance, with proteins, where the biological function is given by the way they fold, which in turn depends on the sequence of aminoacids that compose the protein.

The example we are interested in is also of fundamental biological importance: the genetic sequence contained in the DNA molecule. Theoretical studies of the thermal denaturation (or melting) transition of DNA have resorted to different kinds of models: Ising-like models such as those introduced by Poland and Scheraga [1], or Hamiltonian models including physical interactions, e.g. the model proposed by Peyrard and Bishop [2,3] and other nonlinear

\footnotetext{
a e-mail: saul@mpipks-dresden.mpg.de
}

models [4]. Recently, the inclusion of the genetic sequence in this model has shown the physical importance of functional sites for transcription $[5,6]$ or the theoretical explanation [7] of the experimentally observed [8] denaturation bubbles and cooperative effects in the melting process. In the spirit of the original Peyrard-Bishop model [2], we will see that two simple models proposed in 1981 by Chui and Weeks [9] (similar work was carried at the same time by van Leeuwen and Hilhorst [10]) and Burkhardt [11] for the study of the wetting transition are able, in spite of their simplicity, to characterize the effect of the disorder introduced by the genetic sequence on the melting temperature. In the remainder of the paper, we discuss these models and apply them to the DNA melting problem. From the specific viewpoint of the models considered, our numerical results support the idea that in the ASOS model and in the continuous model the corrections to the critical temperature introduced by disorder are logarithmic as in the RSOS model. From a more general viewpoint, as we will see, in spite of the extreme simplicity of the models, the results are in good semi-quantitative agreement with the experiments. This suggests that in this system the most relevant ingredient is disorder, allowing for modeling other aspects of the problem in a crude but efficient way while still obtaining a generally correct description. We discuss 
this point in more detail in the last section and suggest that it may be useful for models in other contexts. Important conclusions regarding the nature of possible correlations in DNA are also drawn.

\section{Homogeneous Chui-Weeks and Burkhardt models}

In 1981, Chui and Weeks [9] (see also Ref. [10]) proposed a wetting model to study the depinning of an interface from an attractive substrate. The version of their model in which we are interested can be written in terms of a Hamiltonian defined in a one dimensional lattice with periodic boundary conditions:

$$
\mathcal{H}=\sum_{i=1}^{N}\left\{J\left|n_{i+1}-n_{i}\right|-B \delta_{n_{i}, 0}\right\},
$$

where $n_{i}$ are integer variables with positive or zero values, and $N$ is the total number of nodes in the lattice. The variables $n_{i}$ are the distance between the substrate and the interface position at site $i$. The first term in the Hamiltonian can be understood as a surface tension, where $J$ is the coupling constant between nearest neighbors. The second term is a potential which binds the interface to the substrate at $n_{i}=0$, and $B$ is the strength of this attraction. Chui and Weeks showed analytically that this model presents a thermodynamic phase transition (see [12] for a recent review on the existence of one-dimensional phase transitions) between a bound interface at low temperatures and a free one at high temperatures. The critical condition for this transition is given by:

$$
e^{-\beta_{c} B}=1-e^{-\beta_{c} J},
$$

where $\beta_{c}=1 / k_{B} T_{c}$.

In the same year as Chui and Weeks, Burkhardt [11] proposed a model that is the continuous version of the Chui-Weeks discrete model. Its Hamiltonian is:

$$
\mathcal{H}=\sum_{i=1}^{N}\left\{J\left|y_{i+1}-y_{i}\right|+V\left(y_{i}\right)\right\},
$$

where now $0 \leq y_{i}<\infty$ are real variables and the potential $V\left(y_{i}\right)$ has the form:

$$
V\left(y_{i}\right)= \begin{cases}-B, & y_{i} \leq R \\ 0, & y_{i}>R\end{cases}
$$

The model is equivalent to the one proposed by Chui and Weeks, but now the variables are continuous and the substrate attraction has a finite range given by the constant $R$.

Burkhardt showed analytically that this model has the same kind of phase transition than the discrete model. The critical condition now is:

$$
\beta_{c} J R=\left(e^{\beta_{c} B}-1\right)^{-1 / 2} \tan ^{-1}\left[\left(e^{\beta_{c} B}-1\right)^{-1 / 2}\right] .
$$

\section{Disorder}

Forgacs et al. [13] (see also [14]) extended the discrete model in its restricted (RSOS) version, where the restriction $\left|n_{i+1}-n_{i}\right| \leq 1$ is enforced, to include disorder in the potential. The disorder is introduced by letting the potential parameter $B$ in equation (1) be site dependent, uncorrelated and given by a distribution $P(b)$. Depending on whether the partition function is averaged over the disorder (in which case the free energy is $\left.F_{a}=-k_{B} T \log \overline{\mathcal{Z}}\right)$ or the average is done directly over the free energy $\left(F_{q}=-k_{B} T \overline{\log \mathcal{Z}}\right)$ the disorder is said to be annealed or quenched. In the case of annealed disorder the factor $\exp (\beta B)$ which appears at the beginning in the calculation of the solution of the model should be substituted by the average $[13,14]$ :

$$
e^{\widetilde{\beta B}}=\int_{-\infty}^{\infty} \mathrm{d} b P(b) e^{\beta b}
$$

Instead of the restricted version of the model (RSOS) studied in references $[13,14]$, we are interested in the unrestricted version (ASOS) where the difference $\left|n_{i+1}-n_{i}\right|$ can take any positive (or zero) value. To our knowledge, the ASOS model with disorder was not studied in previous references. Following a procedure similar to that for the RSOS case, the new critical condition for the ASOS model with annealed disorder can be found to be:

$$
\left(e^{\widetilde{\beta B}}\right)^{-1}=1-e^{-\beta_{c} J} \text {. }
$$

In the case of a dichotomous disorder which can take a value $B_{1}$ with probability $p$ and a value $B_{2}$ with probability $1-p$, this condition reads:

$$
\frac{1}{p e^{\beta B_{1}}+(1-p) e^{\beta B_{2}}}=1-e^{-\beta_{c} J} .
$$

Nevertheless, the kind of disorder relevant in a DNA molecule is quenched disorder, as the genetic sequence does not change with time. Although in reference [13] the conclusion is reached that the annealed and the quenched cases yield the same critical temperature, later work [14] (reinforced by work on a related model defined on a onedimensional continuum [15]) showed that there is a logarithmic difference between the critical temperature for both types of disorder, which vanishes when the disorder goes to zero. For the values of the parameters we are interested in (see Sect. 5), the difference between the critical temperature predicted for both types of disorder is several orders of magnitude smaller than the temperatures themselves, and hence not measurable by usual means.

Although references $[13,14]$ work for the restricted version of the model, we believe that the same phenomenology appears in the unrestricted one we are using. Subsequently, we will use equation (8) as an approximation for quenched disorder, and we will check afterwards the validity of this approximation by two different numerical approaches: Monte Carlo simulation of the model and numerical evaluation of the transfer matrix. 
As for the Burkhardt model, to our knowledge no attempt has been made to study it with uncorrelated disorder in the potential in the same fashion we have seen for the discrete model. Burkhardt [16] made a study using a corrugated potential, but constructed in a deterministic and periodic way. In reference [17] both the discrete and the continuous models are studied with a somewhat more general kind of disorder in the potential, that also has to be periodic. However, uncorrelated disorder can be studied in the continuous model in the same way that in the discrete one. If the constant $B$ is site dependent and given in each site by a probability distribution $P(b)$, for annealed disorder the factor $\exp (\beta B)$ in Burkhardt's calculation [11] should be substituted by:

$$
e^{\widetilde{\beta B}}=\int_{-\infty}^{\infty} \mathrm{d} b P(b) e^{\beta b} .
$$

This leads to the following critical condition in the presence of annealed disorder:

$$
\beta_{c} J R=\left(e^{\widetilde{\beta_{c} B}}-1\right)^{-1 / 2} \tan ^{-1}\left[\left(e^{\widetilde{\beta_{c} B}}-1\right)^{-1 / 2}\right] .
$$

In the case of a dichotomous disorder as we saw in the discrete model, the factor $\exp (\widehat{\beta B})$ is:

$$
e^{\widetilde{\beta B}}=p e^{\beta B_{1}}+(1-p) e^{\beta B_{2}} .
$$

Again, as in the discrete model, we will assume this formula to be a good approximation also for quenched disorder, and check later this hypothesis by Monte Carlo simulations and numerical evaluation of the transfer operator of the model.

\section{The Peyrard-Bishop model of DNA}

One of the most successful models in biophysics is the Peyrard-Bishop model, which describes in a simplified way (including only one relevant degree of freedom for each base pair, namely the distance between the two bases) the dynamics and statistical mechanics of the DNA molecule. The original formulation by Peyrard and Bishop can be written in terms of the following Hamiltonian [18], disregarding the kinetic term in which we are not interested in this work:

$$
\mathcal{H}=\sum_{i=1}^{N}\left\{\frac{J}{2}\left(y_{i+1}-y_{i}\right)^{2}+V\left(y_{i}\right)\right\},
$$

where $V\left(y_{i}\right)=B\left(e^{-R y_{i}}-1\right)^{2}$ is a Morse potential, $B$ giving the strength of the potential and $R$ the width of the attracting well of the potential. The variables $y_{i}$ can take any real value, and they represent the difference of the actual distance between the two bases in base pair $i$ and their equilibrium distance. The harmonic coupling represents the rigidity of the molecule, due to in part to the stacking interaction between consecutive base pairs. The Morse potential represents the hydrogen bonds between the two bases in a base pair.

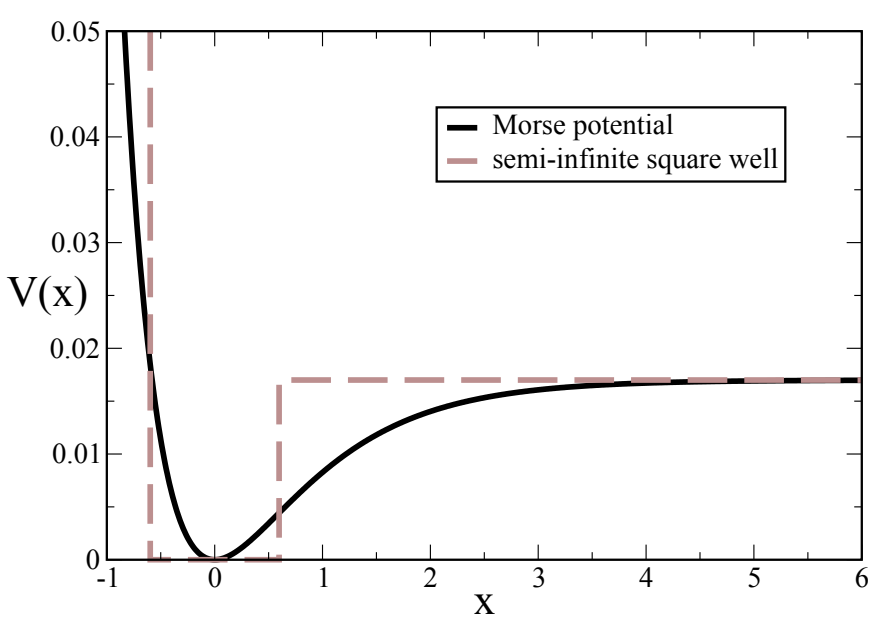

Fig. 1. Comparison between a Morse potential with parameters $B=0.017$ and $R=1.195$ with a semi-infinite square well with the same parameters. The latter has been displaced in order to help the eye.

Note that the Peyrard-Bishop model is formally very similar to the Burkhardt model. The difference in the type of coupling between both of them (absolute value in Burkhardt, harmonic in Peyrard-Bishop) does not introduce qualitative differences. The Morse potential and the semi-infinite square well in the Burkhardt model are qualitatively very similar, as can be seen in Figure 1. These facts make us expect the Burkhardt model to display the same qualitative behavior than Peyrard-Bishop's. This means that we can use the simple Burkhardt model, and the even simpler Chui-Weeks model, its discrete counterpart, to study some aspects of the DNA molecule. In this way we can take advantage from the fact that from these models analytical information can be extracted even when disorder is included.

The dichotomous disorder we introduced above is just the kind of effect that the genetic sequence introduces in the physics of the DNA molecule: the existence of only two kinds of base pairs (adenine-thymine, A-T, and guaninecytosine, G-C) with different number of hydrogen bonds ( 2 for A-T and 3 for G-C) implies that we should take into account two different values for the potential strength $B_{i}$, depending on whether the site $i$ corresponds to an A-T pair or to a G-C pair. For simplicity, in this work we make only the strength of the potential site dependent, keeping its width $R$ constant on all sites.

\section{DNA melting}

The thermal denaturation of DNA is a phase transition where the effect of the genetic sequence can not be ignored [7]. A first (rough) approximation, leaving out the structure in the sequence, is making the hypothesis that a long enough DNA molecule can be treated as an uncorrelated sequence of A-T and G-C pairs, with a fixed concentration of pairs of each type. Then, using the Burkhardt model as a simplification of the Peyrard-Bishop model, 

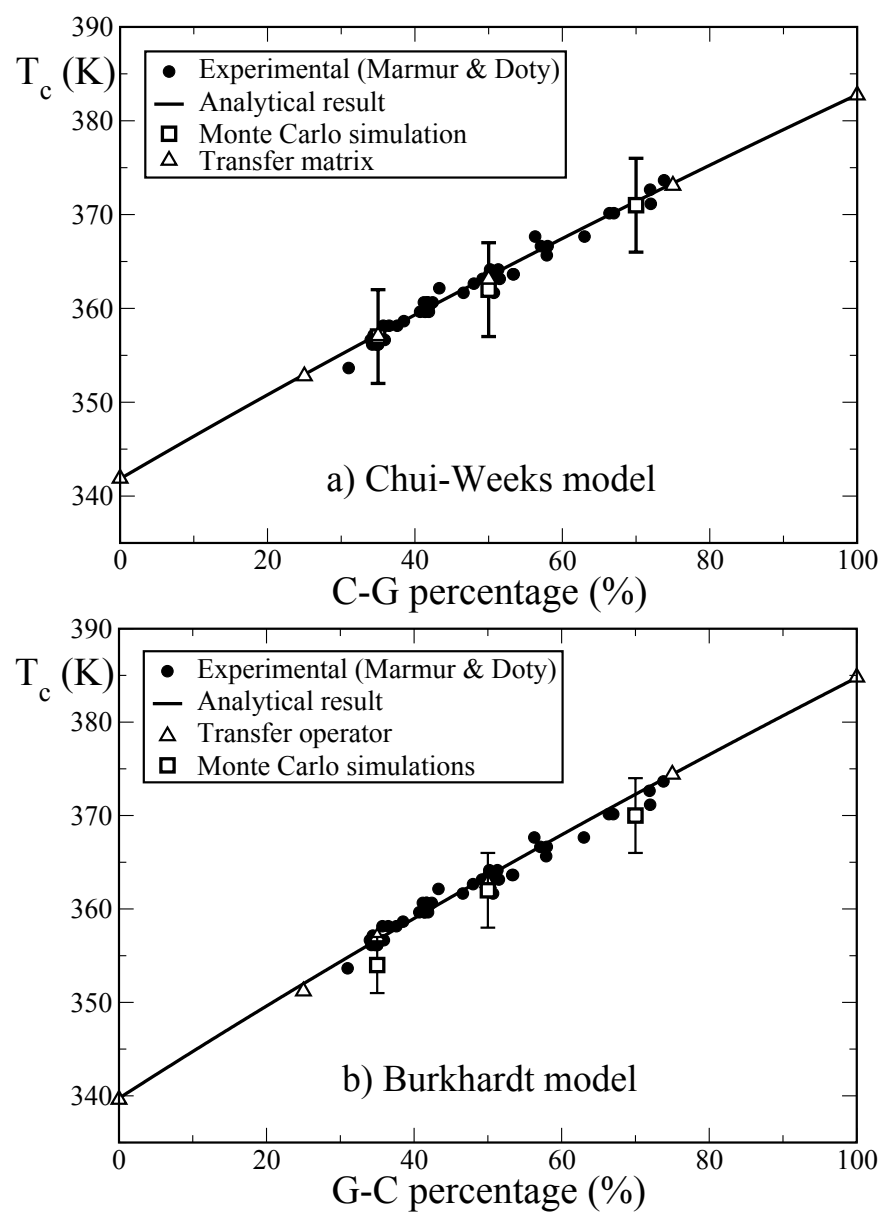

Fig. 2. Comparison of the analytical results for the annealed versions of the models (Eq. (8) in (a) and equation (11) in (b) and the numerical results (Monte Carlo and transfer operator) for the quenched versions. Comparison is made as well with experimental results from reference [19] for the melting temperature as a function of G-C concentration. Note that although they look linear, the predictions of equations (8) and (11) are not linear fits.

we can obtain the critical temperature for a given concentration from equation (10). An even stronger simplification is to use the Chui-Weeks model, in which case it is equation (8) the one that gives the critical temperature. This makes the DNA molecule a useful playground to test experimentally the predictions of including disorder in the wetting models studied. In Figure 2, we show the comparison between experimental data in reference [19] and the prediction of equations (8) and (10), Monte Carlo simulations [20] of the models using the Metropolis algorithm [22] and parallel tempering $[23,24]$, and the numerical evaluation of the transfer operator [25] of the models. The numerical studies confirm that equations (8) and (10) are good approximations for quenched disorder, as we can see by the coincidence of the analytical results for annealed disorder and numerical ones for quenched disorder. This is not strange, since equation (1.7) in reference [14] predicts that, for the discrete RSOS model, the difference between $e^{\widetilde{\beta B_{c}}}$ of the random model and $e^{\beta B_{c}}$ of the pure one is of the order of $10^{-24}$ for the parameters we use, and similar behavior is expected in the ASOS and continuous models we study. The parameters used for both models are: $J=0.03 \mathrm{eV}, B_{G-C}=0.017 \mathrm{eV}, B_{A-T}=0.0132 \mathrm{eV}$ and $R=1.195 \AA$. These values are of the order of magnitude of the parameters accepted for the Peyrard-Bishop model [26], and, as can be seen in the figure, using them the wetting models studied reproduce correctly the experimental dependence of $T_{c}$ on the sequence composition.

\section{Conclusions}

In this work we have revisited two models of wetting, the first introduced by Chui and Weeks and the second by Burkhardt. We have presented the treatment made for the ASOS Chui-Weeks model with disorder and developed an original treatment for the Burkhardt model, finding the critical conditions for both models when the disorder is annealed. Two independent numerical approaches, namely Monte Carlo simulation and evaluation of the transfer operator of the models, confirm that the expressions obtained for annealed disorder are good approximations for the critical conditions with quenched disorder. This suggests that, as in the discrete RSOS model, in the ASOS model and in the continuous model the corrections to the critical temperature introduced by disorder are also logarithmic. Using this two models as simplified versions of the Peyrard-Bishop model of DNA, we have been able to reproduce the dependence on the genetic sequence of the DNA melting temperature.

The agreement between the results of this simple models (one of them is even of discrete nature) and experimental results suggests that, for the class of inhomogeneous models studied here, the effect of the disorder can be characterized using simplified interactions, as long as the disorder itself is properly taken into account. This indicates that a theoretical model where the disorder is introduced in a proper and realistic way can reproduce correctly the effect of disorder, even if the other interactions in the model are not included in a detailed way. Indeed, we have seen that very simple and unrealistic models are enough to display the dependence of the melting temperature of DNA with the concentration of each kind of base pairs. These models do not even have a phase transition of the correct order: it is continuous, while experimentally the melting transition is seen to be first order. A further refinement of the Peyrard-Bishop model, which is more realistic but at the same time more complex, as it includes an anharmonic coupling [3], shows a phase transition of the correct order. However, the description of the effects of the sequence in the general level we have used in this work does not improve much by taking this more realistic model. We have checked, using Monte Carlo Metropolis simulations as those described in [7], that the correct relation between melting temperature and G-C concentration is also recovered by this model [27], which stands as yet another successful experimental test of the Peyrard-Bishop model with the anharmonic coupling, and strengthens our claim 


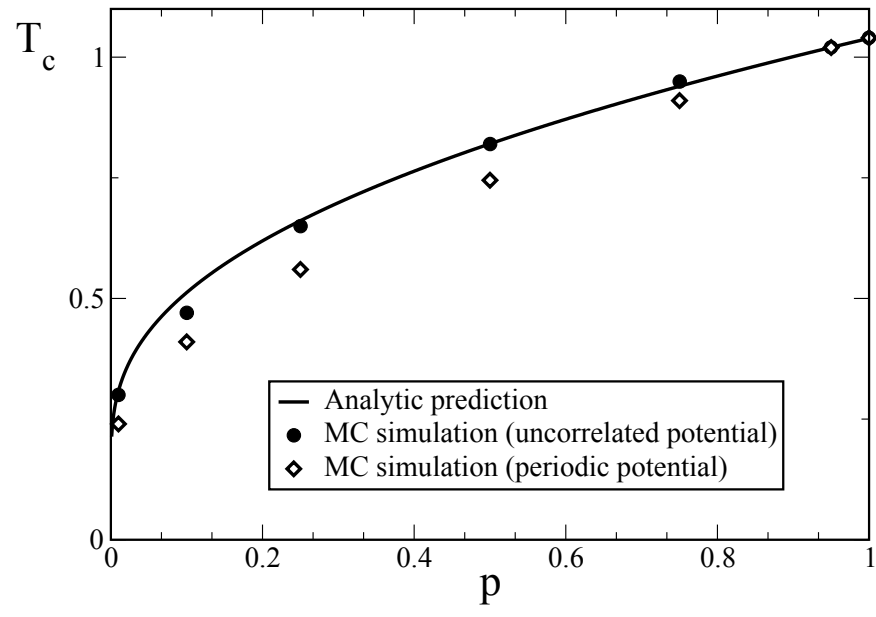

Fig. 3. Critical temperature of the Chui-Weeks model with dichotomous disorder as a function of the fraction of sites $p$ with potential strength $B_{1}$. Parameters: $B_{1}=1, B_{2}=0, J=0.5$.

that the quite simple Chui-Weeks or Burkhardt models where enough to display this phenomenology. Further research about the comparison of other properties as computed from the simple models with the experiments would be necessary to ascertain the degree of usefulness of the models.

This approach, based on choosing disorder as the factor to be modelled more accurately, has proved useful in other contexts other than the one explored here, such as, e.g., protein folding, where a huge huge amount of work has been done using oversimplified models that, nevertheless, have given good results, that even resist quantitative comparison with experiments [28]. Another example can be found in problems involving complex networks, where real problems can be reproduced with simplified interactions or rules granted that the underlying network structure is correctly described [29]. Finally, spin glasses models show how a simple description with complex disorder gives powerful insight of natural phenomena [30]. We believe that other problems in the field of complex systems may benefit from these ideas as, generally speaking, designing simple models as those considered here with a correct description of the disorder may be a very efficient way to obtain rapidly approximate results that may guide further, more detailed studies.

Finally, another conclusion arises from the fact that all the DNA sequences used in reference [19] are natural sequences coming from living organisms. The fact that assuming uncorrelated sequences we have been able to compute the melting temperature means that, in fist approximation, it is reasonable to consider natural that DNA, at least long enough sequences, melts as if it were effectively uncorrelated. In Figure 3 we see a comparison of simulation results for uncorrelated sequences and periodic sequences. We see that the analytic expression works well for the uncorrelated case, but there is a degree of deviation for the periodic one. Therefore, we do not expect our approach to hold for quasi-periodic or highly corre- lated sequences. Interestingly, this implies that if there actually exist correlations in the sequences used in the experiments in [19], they do not affect the melting temperature, which remains unchanged with respect to the melting temperature of purely random sequences. We want to remark again that we have found the same results using the Peyrard-Bishop model with anharmonic coupling [3]. This strengthens the claim that including correlations in the sequence is not crucial for obtaining the correct critical temperature. This observation does not challenge the existence of correlations, even long ranged ones [31], in DNA: it just states that they are weak enough to not have an appreciable effect over the melting temperature of the molecule, although they may be of great biological significance.

This work has been supported by the Ministerio de Ciencia y Tecnología of Spain through grants MOSAICO and NAN200409087-C03-03 and Ingenio Mathematica (i-MATH) (AS) and by Comunidad de Madrid grant SIMUMAT-CM (AS).

\section{References}

1. D. Poland, H.A. Scheraga, J. Chem. Phys. 45, 1456 (1966); D. Poland, H.A. Scheraga, J. Chem. Phys. 45, 1464 (1966); D. Poland, Biopolymers 73, 216 (2004); C. Richard, A.J. Guttmann, J. Stat. Phys. 115, 925 (2004)

2. M. Peyrard, A.R. Bishop, Phys. Rev. Lett. 62, 2755 (1989)

3. T. Dauxois, M. Peyrard, A.R. Bishop, Phys. Rev. E 47, R44 (1993); T. Dauxois, M. Peyrard, Phys. Rev. E 51, 4027 (1995)

4. L.V. Yakushevich, Nonlinear Models of DNA, 2nd edn. (Wiley, 2004)

5. C.H. Choi, G. Kalosakas, K.Ø. Rasmussen, M. Hiromura, A.R. Bishop, A. Usheva, Nucleic Acids Res. 32, 1584 (2004); G. Kalosakas, K.Ø. Rasmussen, A.R. Bishop, C.H. Choi, A. Usheva, Europhys. Lett. 68, 127 (2004)

6. T.S. van Erp, S. Cuesta-López, J.-G. Hagmann, M. Peyrard, Phys. Rev. Lett. 95, 218104 (2005)

7. S. Ares, N.K. Voulgarakis, K.Ø. Rasmussen, A.R. Bishop, Phys. Rev. Lett. 94, 035504 (2005)

8. A. Montrichok, G. Gruner, G. Zocchi, Europhys. Lett. 62, 452 (2003); Y. Zeng, A. Montrichok, G. Zocchi, Phys. Rev. Lett. 91, 148101 (2003); Y. Zeng, A. Montrichok, G. Zocchi, J. Mol. Biol. 339, 67 (2004)

9. S.T. Chui, J.D. Weeks, Phys. Rev. B 23, R2438 (1981)

10. J.M.J. van Leeuwen, H.J. Hilhorst, Physica A 107, 319 (1981)

11. T.W. Burkhardt, J. Phys. A 14, L63 (1981)

12. J.A. Cuesta, A. Sánchez, J. Stat. Phys. 115, 869 (2004)

13. G. Forgacs, J.M. Luck, Th.M. Nieuwenhuizen, H. Orland, Phys. Rev. Lett. 57, 2184 (1986); J. Stat. Phys. 51, 29 (1988)

14. B. Derrida, V. Hakim, J. Vannimenus, J. Stat. Phys. 66, 1189 (1992)

15. S.M. Bhattacharjee, S. Mukherji, Phys. Rev. Lett. 70, 49 (1993); S. Mukherji, S.M. Bhattacharjee, Phys. Rev. E 48, 3483 (1993)

16. T.W. Burkhardt, J. Phys. A 31, L549 (1998) 
17. P. Nowakowski, M. Napiórkowski, J. Phys. A 38, 5885 (2005)

18. T. Dauxois, M. Peyrard, A.R. Bishop, Phys. Rev. E 47, $684(1993)$

19. J. Marmur, P. Doty, J. Mol. Biol. 5, 109 (1962)

20. Monte Carlo simulations have been done following the procedure described in [21]. $5 \times 10^{5}$ tries of replica exchange are used, and between them each replica is simulated a number of Monte Carlo steps equal to the energy autocorrelation time at the replica's temperature, determined during previous simulations used for equilibration

21. S. Ares, J.A. Cuesta, A. Sánchez, R. Toral, Phys. Rev. E 67, $046108(2003)$
22. N. Metropolis, A.W. Rosenbluth, M.N. Rosenbluth, A.H. Teller, E. Teller, J. Chem. Phys. 21, 1087 (1953)

23. M.E.J. Newman, G.T. Barkema, Monte Carlo Methods in Statistical Physics (Oxford University, Oxford, 1999)

24. Y. Iba, Int. J. Mod. Phys. C 12, 623 (2001)

25. S. Ares, A. Sánchez, Phys. Rev. E 70, 061607 (2004)

26. A. Campa, A. Giansanti, Phys. Rev. E 58, 3585 (1998)

27. S. Ares, A. Sánchez, unpublished data

28. B.H. Park, M. Levitt, J. Mol. Biol. 249, 493 (1995)

29. M.E.J. Newman, SIAM Rev. 45, 167 (2003)

30. K. Binder, A.P. Young, Rev. Mod. Phys. 58, 801 (1986)

31. W. Li, Computers Chem. 21, 257 (1997) 